\title{
RedFace: Um Sistema de Reconhecimento Facial para Identificação de Estudantes em um Ambiente Virtual de Aprendizagem
}

Fábio Abrantes Diniz - PPgCC - UERN/UFERSA - fabio.abrantes.diniz@ gmail.com Francisco Milton Mendes Neto - PPgCC - UFERSA - miltonmendes@ufersa.edu.br

Francisco das Chagas Lima Júnior - PPgCC - UERN - limajunior@uern.br

Laysa Mabel de O. Fontes - PPgCC - UERN/UFERSA - laysa@ ufersa.edu.br

Resumo. Atualmente, a maioria dos Ambientes Virtuais de Aprendizagens (AVAs) não dispõe de mecanismos eficientes que possam identificar os alunos tanto no acesso ao sistema quanto durante o desenvolvimento de suas atividades. Em geral, este tipo de sistema adota como prática de segurança a utilização de mecanismos de autenticação por login e senha. O uso deste tipo simples de autenticação aumenta a vulnerabilidade a fraudes no aspecto relativo à identidade daquele que realiza o curso. Para preencher esta lacuna, este trabalho desenvolve $e$ aplica um sistema de reconhecimento facial baseado em técnicas de Análise de Componentes Principais e Autofaces em um AVA, e investiga o seu desempenho na identificação do aluno durante toda a realização do curso em um $A V A$.

Palavras-chave: Faces, Ambiente Virtual de Aprendizagem, Moodle, Alunos, Identificação.

\section{RedFace: A Face Recognition System for Students Identification in a Virtual Learning Environment}

\begin{abstract}
Currently, most of Virtual Learning Environments (VLEs) has no efficient mechanisms that can identify students accessing the system and during the development of their activities. In general, this type of system adopts the use of login and password as security mechanism for authentication. The use of this simple type of authentication increases vulnerability to fraud in relation to the identity of who carries the course. For fulfill this gap, this paper develops and applies a face recognition system based on techniques of Principal Component Analysis and Autofaces in a $A V A$, and investigates their performance in identifying the student during the entire course in a VLE.
\end{abstract}

Keywords: Face, Virtual Learning Environments, Moodle, Student, Identifying. 


\section{Introdução}

A avaliação do aluno em cursos online se mostra desafiadora para professores e instituições envolvidas nessa modalidade de ensino. É difícil estabelecer um sistema confiável para garantir que o aluno que participa das avaliações é o mesmo que vai receber a certificação ou diplomação. Logo, a identificação de alunos em Ambientes Virtuais de Aprendizagens (AVAs) é um fator crítico para a segurança na realização de um curso de ensino a distância (Fiorese; Tarouco, 2006).

Existe uma variedade de métodos para a identificação de usuários, os quais são utilizados no controle de acesso em aplicações comerciais (Marais et al, 2006). Porém, nos AVAs, essas técnicas de identificação não estão sendo muito utilizadas, pois as restrições de ordem econômica e operacional são diferentes (Marais et al, 2006). Em geral, alguns destes ambientes adotam a utilização de mecanismos de identificação por login e senha como prática de segurança. $\mathrm{O}$ uso deste tipo simples de autenticação aumenta a vulnerabilidade a fraudes, tanto no acesso ao sistema quanto durante a participação do aluno nas atividades do curso, pois outra pessoa pode substituir facilmente o indivíduo que deveria ser avaliado no AVA acessado (Rabuzin, 2006).

$\mathrm{O}$ avanço da tecnologia biométrica, aliada à redução dos preços dos equipamentos, fez com que surgissem várias pesquisas da biometria em ambientes Web (Almeida, 2003). Logo, os sistemas de reconhecimento biométrico, quando bem projetados e utilizados, tornam a identificação de usuários em sistemas online mais segura e viável, em particular nos AVAs. Baseado nisso, este trabalho propõe um sistema de reconhecimento facial, denominado RedFace, aplicado a um AVA. Esse AVA é composto por agentes de software que auxiliam na aplicação correta da teoria da Aprendizagem Baseada em Problema (Problem-Based Learning - PBL) (Melo-Solarte; Baranauskas, 2008).

O RedFace adiciona a funcionalidade de autenticação biométrica ao AVA. Monitora um estudante, capturando imagens através de webcam, bem como detecta a face do estudante na imagem e a identifica dentre outras faces cadastradas no sistema. Para isso, utilizou-se o algoritmo Viola-Jones (Viola; Jones, 2004) para a detecção da face, e as técnicas de Análise de Componentes Principais (PCA) (Stan; Anil, 2011, Romdhani, 1996) e Eigenfaces (Stan; Anil, 2011, Romdhani, 1996, Jiangsheng, 2002) no processo de extração de características da face. Na classificação das imagens, foi usado o classificador K-Nearest Neighbors (K-NN) (Jiangsheng, 2002). Os resultados parciais obtidos mostram que o RedFace pode ser aplicado com sucesso na confirmação da identidade dos alunos durante todo o período de realização de um curso em um AVA.

Este trabalho está organizado da seguinte forma: a Seção 2 descreve o AVA baseado em agentes no qual o sistema de reconhecimento facial desenvolvido foi aplicado; a Seção 3 apresenta o sistema de reconhecimento facial proposto, mostrando suas etapas de implementação; a Seção 4 discute os trabalhos relacionados; a Seção 5 apresenta uma análise dos resultados; e, por fim, na Seção 6, são apresentadas as considerações finais e as propostas de trabalhos futuros.

\section{Ambiente Virtual de Aprendizagem Baseado em Agentes de Apoio à PBL}

O AVA baseado em agentes de apoio à PBL, no qual o RedFace foi aplicado, está esquematizado na Figura 1. Ele utiliza a arquitetura cliente-servidor padrão do Moodle (França; Soares, 2011). No lado cliente, são executadas as páginas em PHP e o Agente 
3

Pedagógico Animado (AgPA), os quais solicitam serviços ao servidor da aplicação, através da Internet. No lado servidor localiza-se o sistema gerenciador de banco de dados (SGBD), onde são armazenados todos os dados referentes aos cursos, aos alunos cadastrados (inclusive suas imagens faciais), além de quatro tipos de agentes: Agente Detector de Problemas (AgDP), Agente Formador de Grupos (AgFG), Agente Recomendador (AgR) e Agente DF (Directory Facilitator).

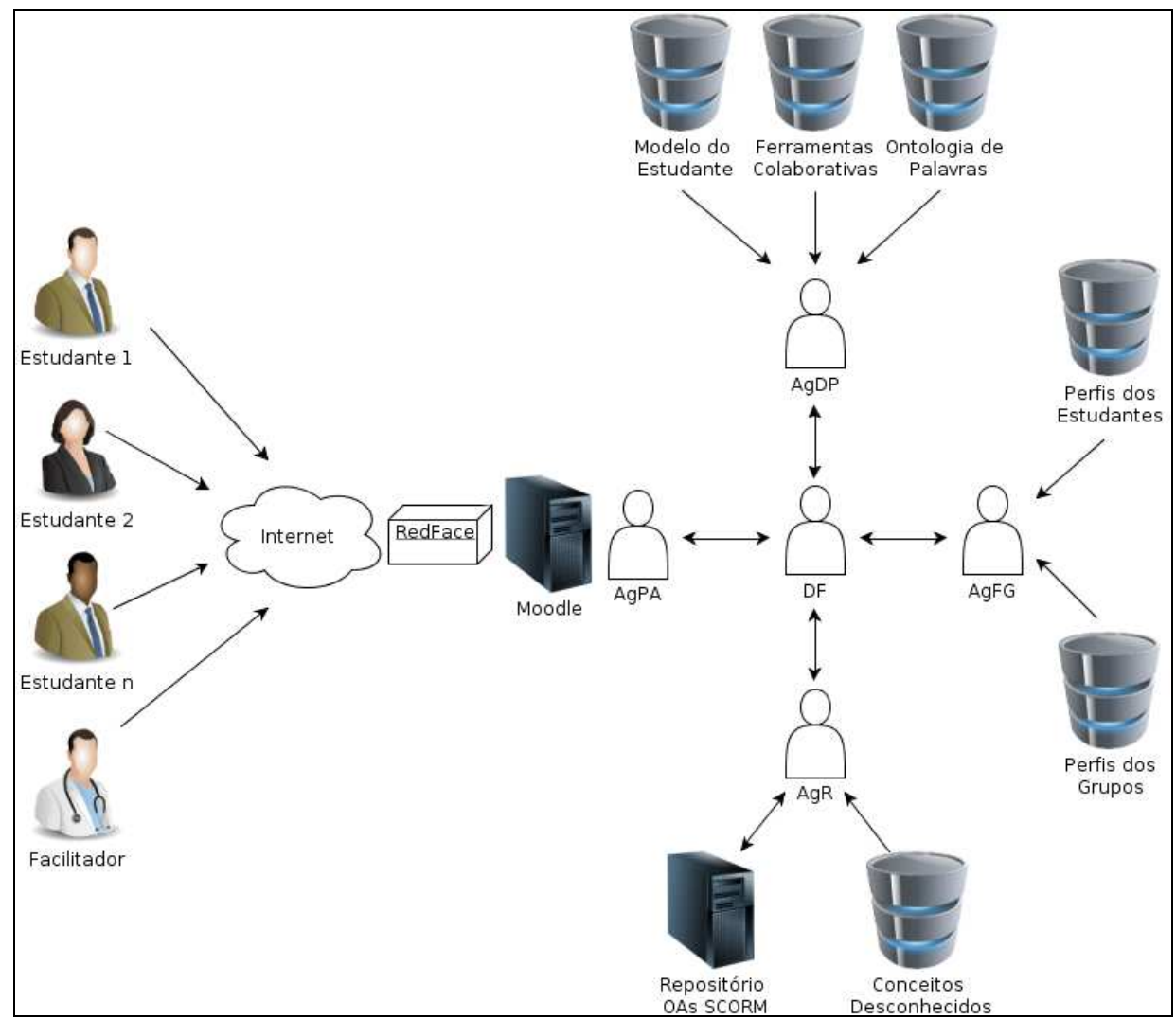

Figura 1. Arquitetura de apoio à PBL

O meio padrão de acesso ao Moodle (França; Soares, 2011) se dá através de login e senha, o que não garante a veracidade da identidade do aluno. Como pode ser visto na Figura 1, o aluno deve, inicialmente, se autenticar no Moodle (Kumar et al, 2011), utilizando o RedFace (mais detalhes na Seção 3), e acessar algum dos cursos nos quais esteja matriculado. O RedFace é um sistema que identifica o aluno, via webcam, tanto no acesso como durante a utilização do AVA, capturando, em tempos predefinidos, a imagem facial do aluno. Desta forma, o RedFace registra a identidade de quem está realizando as atividades pedagógicas e avaliações no processo da PBL, reforçando assim a garantia da participação do aluno.

Esta arquitetura foi criada com o intuito de auxiliar na aplicação correta da teoria de aprendizagem PBL (Melo-Solarte; Baranauskas, 2008). Os agentes, anteriormente citados, foram criados com o objetivo de aperfeiçoar a aplicação da PBL nos seguintes aspectos: detecção de alunos passivos, detecção de conversações fora do contexto, formação de grupos e recomendação de objetos de aprendizagem (OAs) sensível ao contexto do aluno. Esses agentes trabalham colaborativamente com o objetivo de detectar e corrigir situações indesejadas, melhorando o processo de aprendizagem. A descrição do comportamento do sistema multiagente não é o foco deste artigo. 


\section{4}

\section{Sistema de Reconhecimento Facial}

O sistema de reconhecimento facial proposto consiste basicamente das seguintes etapas: (a) aquisição das imagens; (b) pré-processamento; (c) extração de características; e (d) classificação e verificação (Stan;Anil, 2011). A arquitetura do RedFace é apresentada na Figura 2.

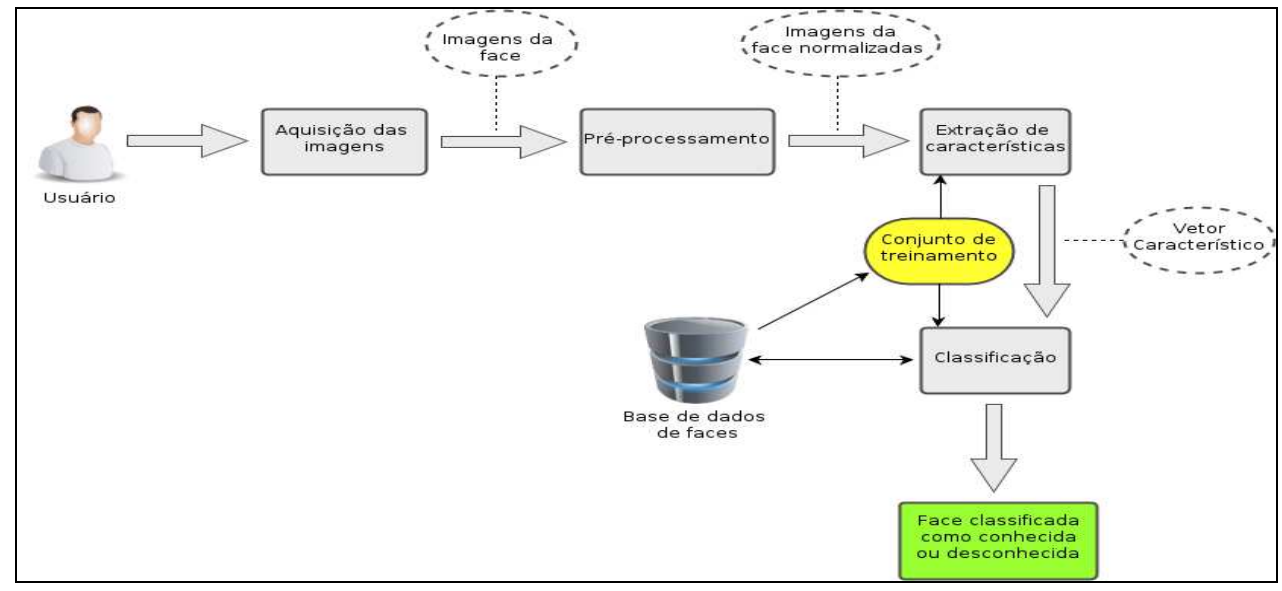

Figura 2. Arquitetura do Sistema de Reconhecimento Facial

O início do processo de reconhecimento de face se dá a partir do módulo de aquisição da imagem. Através da webcam, o sistema captura a imagem da face do aluno a qual será utilizada como entrada para o sistema de reconhecimento facial. Nesse módulo, é utilizado o algoritmo para detecção de face Viola-Jones (Viola; Jones, 2004). Este algoritmo tenta encontrar, em uma imagem, características que codificam alguma informação da classe a ser detectada. Para tal tarefa, são usadas as chamadas características de Haar, responsáveis por codificar informações sobre a existência de contrastes orientados entre regiões da imagem. As imagens são então amostradas quadro a quadro e as faces são detectadas e extraídas usando o algoritmo Viola-Jones, disponível na biblioteca OpenCV (OpenCV, 2011).

O próximo passo é o pré-processamento da imagem. No módulo responsável por esta tarefa, as imagens são normalizadas e corrigidas para melhorar o reconhecimento da face. As fotos passam por correções na dimensão (todas foram formatadas para 120x90, $32 \times 24$ e 12x9), na resolução (300 dpi) e na iluminação (é utilizada a mesma em todas as fotos) e foram convertidas para escala de cinza.

Após o pré-processamento, a imagem da face normalizada serve de entrada para o módulo de extração de características, objetivando encontrar as principais características a serem utilizadas para a classificação. Cada imagem representante do indivíduo é transformada em uma matriz de tamanho $\mathrm{w} x \mathrm{~h}$, onde $\mathrm{w}$ e h são, respectivamente, os números de pixels referentes à largura e à altura da imagem (Romdhani, 1996). Cada valor do pixel da imagem corresponde a uma componente do vetor.

Devido à alta dimensionalidade dos vetores, é utilizada, nesta etapa, a técnica de PCA, visando reduzir a dimensionalidade de uma imagem a fim de diminuir o custo computacional e melhorar a precisão do classificador. O PCA tenta construir um pequeno conjunto de componentes que resumem os dados originais, preservando os componentes mais significantes (Kshirsagar et al, 2011). O PCA é baseado nos 
autovetores e autovalores da matriz de covariância dos dados, onde o objetivo é encontrar um conjunto de vetores ortonormais que melhor descreve a distribuição dos dados de entrada (Agarwal et al, 2010). Este conjunto de vetores ortonormais são então usados para determinar a identidade do aluno. Se o espaço característico contiver somente as características mais visíveis, o classificador será mais eficiente na classificação e ocupará menos memória (Agarwal et al, 2010).

Juntamente com a técnica PCA, é utilizada a técnica do Eigenfaces, detalhada na Subseção 3.2. O algoritmo Eigenfaces objetiva fornecer um conjunto de vetores de distribuições probabilísticos, para resolver o problema da detecção de padrões em imagens. O seu fundamento básico é a utilização desses vetores para gerar uma informação matemática do rosto de um ser humano para sua futura identificação.

Com a ajuda de um classificador de padrões, as características extraídas da imagem da face são comparadas com as amostras do conjunto de treinamento de face. A imagem da face é então classificada como conhecida ou desconhecida. Neste trabalho, foi analisado o algoritmo de reconhecimento de padrão K-NN (K-Nearest Neighbors) (Jiangsheng, 2002), detalhado na Subseção 3.1.

O outro módulo apresentado na Figura 2 é o conjunto de treinamento. Ele visa encontrar características apropriadas para a representação de padrões de entrada, sendo o classificador treinado para particionar o espaço de características, formando padrões de todas as classes existentes. Como pode ser visto na Figura 2, a arquitetura proposta utiliza uma base de dados de faces. Para validação do trabalho, a base de dados foi alimentada com 1280 imagens de um total de 64 pessoas (alunos) cadastradas no AVA. A Figura 3 (A) ilustra algumas faces armazenadas na base de dados utilizada. Cada indivíduo integrante da base de dados é representado por 20 imagens nas seguintes posições: 4 imagens olhando diretamente para a câmera, 4 imagens olhando 45 graus à esquerda da câmera, 4 imagens olhando 45 graus à direita da câmera, 4 imagens olhando 90 graus à esquerda da câmera e 4 imagens olhando 90 graus à direita da câmera, como pode ser visto na Figura 3 (B). Dessas 20 imagens, 5 (cada uma de uma posição) compõem o conjunto de treinamento do sistema e as 15 restantes compõem o conjunto de teste.

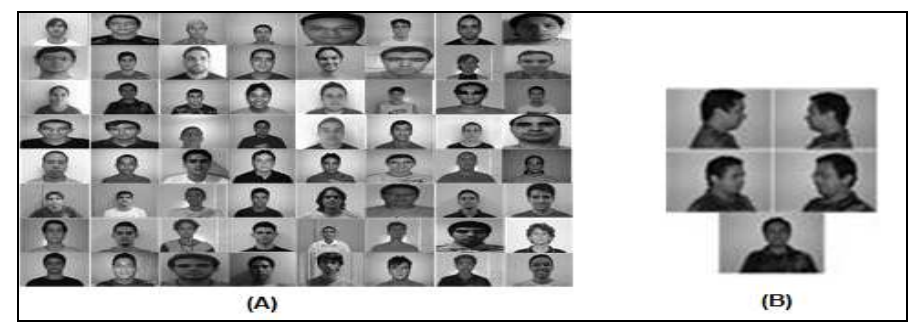

Figura 3. (A) Imagens de faces da base de dados, (B) Esquema de posicionamento da face.

\subsection{Classificador K-Nearest Neighbors}

O K-NN é um dos algoritmos de classificação mais utilizados na área de aprendizagem de máquina (Jiangsheng, 2002). É baseado na procura dos $k$ vizinhos mais próximos do padrão de teste (Jiangsheng, 2002). A busca pela vizinhança é feita utilizando uma medida de distância. Nos experimentos desenvolvidos neste trabalho, além da medida Euclidiana, foram utilizadas a distância de Manhattan (Vadivel et al, 2003) e a Euclidiana normalizada (Jiangsheng, 2002). Além disso, todos os experimentos com K- 
6

NN foram realizados com peso pela distância. Dessa maneira, o padrão é classificado de acordo com a soma dos pesos dos $k$ vizinhos - o peso é o inverso das distâncias. $\mathrm{O}$ padrão é dito pertencer à classe que apresentar a maior soma dos pesos entre os entre os $k$ vizinhos mais próximos (Jiangsheng, 2002). O $k$ escolhido será o que proporcionar menor quantidade erros na classificação das imagens.

\subsection{Eigenfaces}

O método Eigenfaces busca um conjunto de características que não depende das formas geométricas da face (olhos, nariz, orelhas e boca), utilizando toda a informação da representação facial (Kshirsagar et al, 2011). Seu funcionamento é similar ao funcionamento do PCA. Entretanto é utilizada uma leve otimização para reduzir a matriz de covariância, reduzindo o processamento necessário para fazer o cálculo de seus autovetores e autovalores.

Baseadas na Teoria da Informação, as Eigenfaces buscam identificar um pequeno número de características que são relevantes para diferenciar uma face de outras faces. Essas características podem ser analisadas apenas com a variação dos valores assumidos pelos pixels, em um conjunto de imagens de faces.

Os autovetores da matriz de covariância, de um conjunto de imagens de faces, descrevem a variação dos pixels em uma região diferente das imagens, ou seja, significa que cada autovetor descreve a variação dos pixels associados a diferentes características faciais (Turk, 1991).

Depois de encontrar os autovetores, as imagens de faces são projetadas em um espaço de características ("espaço de faces"), que melhor representa a variação entre faces conhecidas. Este espaço é definido pelas Eigenfaces, que são os autovetores do conjunto de faces. O reconhecimento de uma face é realizado pela sua projeção no subespaço gerado pelas Eigenfaces e em seguida pela comparação da posição obtida com a posição de indivíduos conhecidos (Turk, 1991). A sequência dos procedimentos da geração das Eigenfaces, proposta por este trabalho, teve como base o trabalho dos autores Cheon e Kim (Cheon; Kim, 2008). A Figura 4 apresenta a face média do conjunto de treinamento (Figura 3) utilizado neste trabalho e algumas Eigenfaces calculadas para o conjunto de imagens de treino de exemplo.

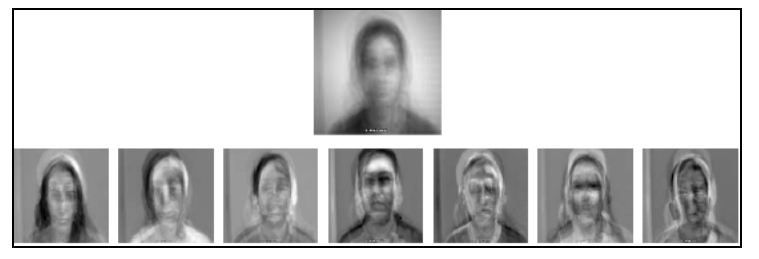

Figura 4: Exemplo de face média (acima) e Eigenfaces mais importantes.

\section{Trabalhos Relacionados}

O reconhecimento facial é um dos métodos biométricos de reconhecimento de padrão que utiliza características particulares da face. Este método apresenta várias vantagens sobre outras tecnologias biométricas (Stan; Anil, 2011). A investigação nesta área tem sido conduzida há mais de 30 anos e, como resultado, o estado atual da tecnologia de reconhecimento facial está bem avançado (Stan; Anil, 2011). 
De um modo geral, os sistemas de reconhecimento facial consistem das seguintes etapas (Stan; Anil, 2011): (i) detecção, que é responsável pela detecção da área da face. Isto pode ser feito observando atributos visuais que distinguem uma face humana de outros objetos; (ii) extração de características, que fornecem informações eficazes e úteis para distinguir entre as faces de pessoas diferentes e estáveis em relação às variações geométricas e fotométricas; e (iii) classificação, que consiste em identificar uma pessoa, comparando sua face com a de outras pessoas cujas imagens estejam armazenadas em um banco de imagens. A ampla disponibilidade de computadores mais potentes, e de baixo custo, criou um grande interesse em pesquisa em processamento automático de imagens digitais e vídeos em uma série de aplicações, incluindo autenticação biométrica, vigilância, saúde, educação, gestão multimídia, entre outras (Stan; Anil, 2011).

$\mathrm{Na}$ educação, poucos trabalhos abordando reconhecimentos faciais foram encontrados. Um dos trabalhos relacionados foi proposto pelos autores Rolim e Bezerra (Rolim; Bezerra, 2008). Neste trabalho, os autores apresentam um sistema de identificação de rosto para ser usado em um AVA com arquitetura cliente-servidor. Esse sistema monitora os alunos remotos através de uma webcam, habilitando a segurança digital na identificação de alunos online no AVA. Segundo Rolim e Bezerra (2008), o sistema de reconhecimento facial utiliza uma técnica baseada em coeficientes DCT (Discrete Cosine Transform).

Apesar da boa precisão verificada no sistema apresentado em (Rolim; Bezerra, 2008), o resultado não pode ser comparado em relação ao trabalho proposto, pois foram feitos testes com imagens de apenas 14 pessoas, cada uma com três poses, enquanto que neste trabalho foram feitos testes com imagens de 64 pessoas, cada uma com cinco poses. Além disso, o trabalho proposto por Rolim e Bezerra (2008) não utiliza detecção e extração de face, o que pode acarretar aumento do tempo de processamento e diminuição da precisão. No presente trabalho, é proposta a detecção da face com a técnica de Viola-Jones (Viola;Jones, 2004), que diminui os efeitos do fundo da imagens, além da técnica de Eigenfaces (Turk, 1991), que restringe as informações desnecessárias.

\section{Experimentos e Resultados}

As simulações foram feitas em cima do monitoramento dos alunos remtos cadastrados no AVA através de uma webcam, habilitando a segurança digital na identificação de alunos online no AVA. Os experimentos foram conduzidos com a mudança das técnicas e dos seus parâmetros. Todos os experimentos foram executados de forma automática e exaustiva, de modo que foi analisado somente o melhor resultado da taxa de acerto para o conjunto de testes (número de acertos dividido pelo número total de testes), utilizando o 1-NN (primeiro vizinho mais próximo) e distância Euclidiana normalizada. Inicialmente, foi executado o redimensionamento (resize) das imagens, resultando em três dimensões diferentes: 120x90 - tamanho normal das imagens; 32x24 - tamanho aproximado de um ícone (Hyun-Chul; Se-Young, 2012) e 12x9 - melhor resultado dos testes automáticos.

Em seguida, os testes foram desenvolvidos tentando encontrar o melhor número de características restantes após o PCA (número de autovetores do Eigenfaces). Foram utilizados valores de 15 a 120 e, em caso de empate (mesma taxa de acerto para número de características diferentes), o menor número de características foi escolhido como 
melhor por garantir menor custo computacional. $\mathrm{O}$ resultado obtido para cada dimensão foi: $120 \times 90$ - 17 características; 32×24 - 19 características e $12 \times 9$ - 20 características.

A Figura 5 apresenta os resultados da taxa de acerto de uma simulação durante a realização de um curso com os alunos cadastros no AVA. Foi analisado segundo as seguintes configurações: as três dimensões das imagens; número de características variando de 15 a 20 (não são mostrados os testes completos por serem muito extensos e por não terem apresentado bons resultados); e $k$ no intervalo de 1 a 10 (k-NN); fazendo uso das três medidas de distância já citadas.

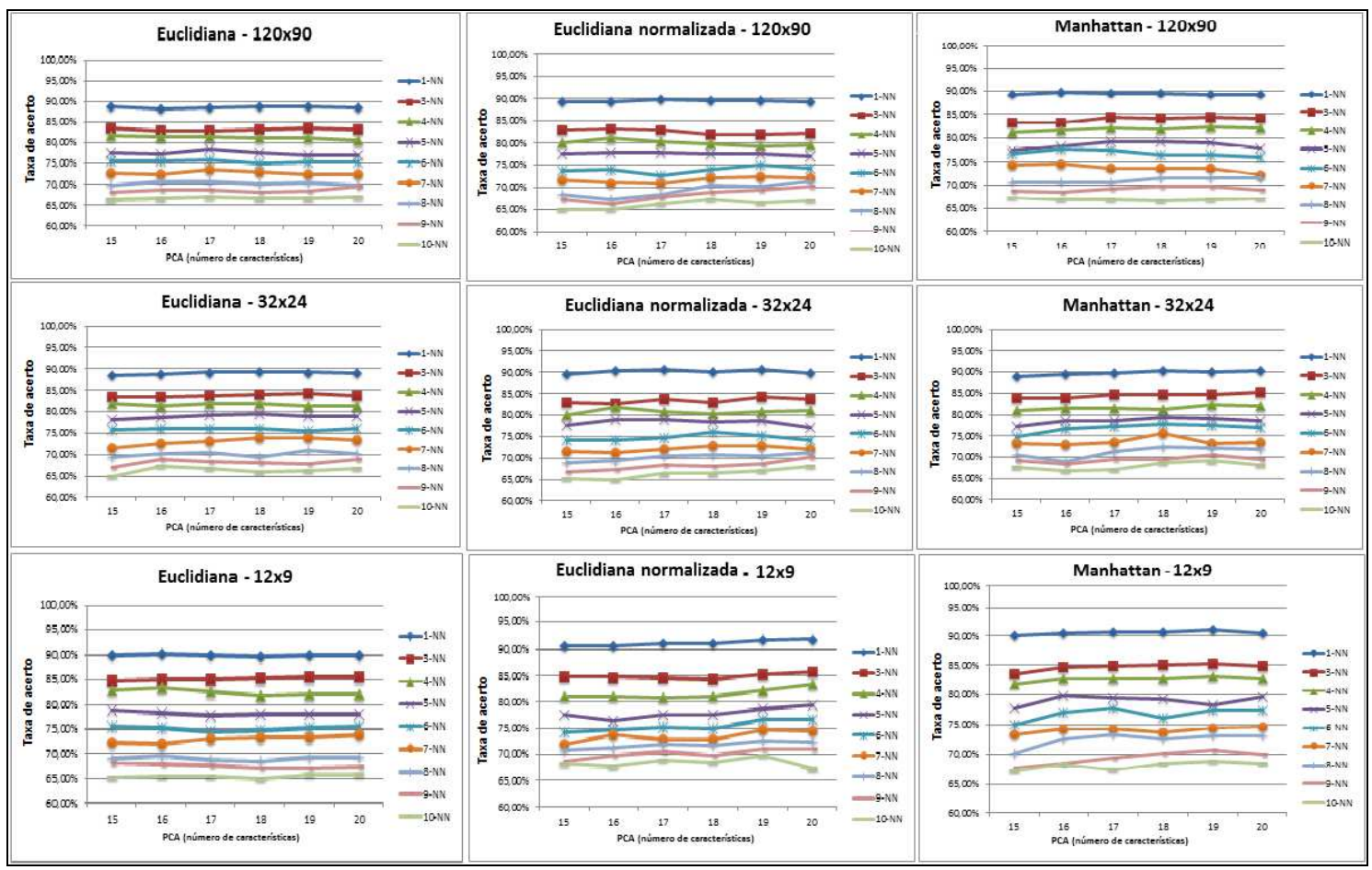

Figura 5. Resultados da taxa de classificação para as imagens com dimensões 120x90, $32 \times 24$ e $12 \times 9$.

\subsection{Discussão dos Experimentos}

$\mathrm{Na}$ Figura 5, nos gráficos relacionados às imagens de face de dimensão $120 \mathrm{x} 90$, verifica-se a maior taxa de acerto $(89,9 \%)$, usando o $k=1$, número de autovetores igual a 17 e distância Euclidiana normalizada. A menor taxa de acerto é observada quando se usa o $k=10$ e distância Euclidiana normalizada. É importante destacar que, para os outros valores de $k$, os melhores resultados são encontrados utilizando a distância Manhattan (Vadivel et al, 2003). Os gráficos das faces de dimensão 32x24 (Figura 5) mostram que a maior taxa de acerto $(90,63 \%)$ é observada usando o $k=1$, número de autovetores igual a 19 e distância Euclidiana normalizada. A menor taxa de acerto é observada quando se usa o $k=10$ e distância Euclidiana. É importante registrar também que, para os outros valores de $k$, os melhores resultados são encontrados utilizando a distância Manhattan. Além disso, há uma uniformidade dos melhores resultados, se encontrando entre os valores característicos do PCA variando entre 18 e 20.

Já nos gráficos das faces de dimensão 12x9 (Figura 5), verifica-se que a maior taxa de acerto (91,98\%) foi observada usando o $k=1$, número de autovetores igual a $20 \mathrm{e}$ distância Euclidiana normalizada. A menor taxa de acerto é observada quando se usa o 
$k=10$ e distância Euclidiana. Para os outros valores de $k$, os melhores resultados são encontrados utilizando a distância Euclidiana normalizada, com os melhores resultados se encontrando entre os valores característicos do PCA, variando entre 19 e 20. Concluímos, com os resultados obtidos nas três dimensões apresentadas na Figura 5, que o aumento dos valores de $k$ implica na diminuição da precisão da classificação.

Além disso, quanto menor a dimensão do pixel, maior é a resolução espacial da imagem. Quanto maior a resolução espacial da imagem, maior será a definição e o nível de detalhe da imagem. Uma imagem com resolução baixa ficará borrada e com menos detalhes, diminuindo o reconhecimento de padrão. Isto favoreceu os melhores resultados encontrados nas imagens de dimensão $12 \times 9$.

\section{Considerações Finais e Trabalhos Futuros}

Neste trabalho, são tratados problemas para o reconhecimento facial na identificação do aluno. A abordagem, utilizando Eigenfaces e PCA, provou ter capacidade de fornecer as características significativas e reduzir o tamanho de entrada para os algoritmos de classificação, melhorando o reconhecimento da face para identificação do aluno. $O$ algoritmo de detecção de faces, portanto, mostrou-se eficiente tanto na taxa de acertos quanto no tempo de processamento. No entanto, esta abordagem mostrou-se ser sensível em imagens com condições de iluminação não controladas.

Como contribuição, este trabalho apresentou uma alternativa biométrica aos métodos atuais de autenticação de indivíduos em um AVA, normalmente baseados em senhas. Concluímos, a partir dos resultados desse trabalho, que a autenticação biométrica pode ser usada para garantir a identidade do aluno que está acessando um AVA remotamente. Experimentalmente, verificamos que esta abordagem de reconhecimento facial, utilizando PCA, Eigenfaces e a técnica de classificação K-NN, apresentou bons resultados. O melhor resultado obtido de testes foi atingido com a técnica K-NN, em imagens de dimensões $12 \times 9$ e utilizando números de 20 características resultantes do PCA, resultando uma taxa de reconhecimento de aproximadamente $92 \%$. Como

trabalhos futuros, pretende-se utilizar a abordagem de PCA e Eigenfaces em uma análise de algoritmos de aprendizado, tais como: Redes Neurais e Redes Bayesianas. Devido aos bons resultados obtidos neste trabalho, também está sendo projetado um sistema de reconhecimento de expressões faciais, utilizando a abordagem de PCA e Eigenfaces e o algoritmo K-NN como classificador das emoções faciais.

\section{Referências}

AGARWAL, M.; Agrawal, H.; Jain, N. Kumar, M. Face Recognition UsingPrinciple Component Analysis, Eigenface and Neural Network, International Conference on Signal Acquisition and Processing. ICSAP 10, vol., no., pp. 310-314, 9-10, 2010.

ALMEIDA, M. E. B. Tecnologia e educação a distância: abordagens e contribuições dos ambientes digitais e interativos de aprendizagem, in Reunião Anual da ANPED, Poços de Caldas, MG,2003.

CHEON, Y.; Kim, D. A Natural Facial Expression Recognition Using DifferentialAAM and k-NNS, Tenth IEEE International Symposium on Multimedia, vol., no., pp.220-227, 15-17, 2008. 
10

FIORESE, M.; Tarouco, L. M. R. Uma Proposta de Autenticação de Usuários para Ensino a Distância. 18 18 $^{\circ}$ Simpósio Brasileiro de Redes de Computadores. Instituto de Informática da UFRGS. Porto Alegre - RS- Brasil, 2006

FRANÇA, A. B.; Soares J. M. Sistema de apoio a atividades de laboratório de programação via Moodle com suporte ao balanceamento de carga, in Anais do Simpósio Brasileiro de Informática na Educação (SBIE), Aracaju, 2011.

HYUN-CHUL C.; Se-Young O. Multi-Face Detection on static image using Principal Component Analysis. ICCAS. Disponível em: < http://isl.postech.ac.kr >. Acesso em: Março 2012.

JIANGSENG, Y. Method of k-Nearest Neighbors. Institute of Computational Linguistics, Peking University, China, 2002.

KSHIRSAGAR, V. P.; Baviskar, M. R.; Gaikwad, M. E. Face recognition using Eigenfaces, Computer Research and Development (ICCRD), 3rd International Conference on, vol. 2, no., pp. 302-306, 11-13,2011.

KUMAR, S., Gankotiya, A. K. e Dutta, K. A Comparative Study of Moodle with other e-Learning Systems. In: International Conference on Electronics Computer Technology - ICECT, 3. Kanyakumari: IEEE, 2011.

MARAIS, E.; Argles D.; von Solms B. Security issues specific to e-assessments, 8th Annual Conference on WWW Applications, 2006.

MELO-SOLART, D. S. ; Baranauskas M. C. C. Uma Abordagem para EaD Baseada em Resolução de Problemas, in Anais do Simpósio Brasileiro de Informática na Educação (SBIE), Fortaleza, 2008.

OPENCV Open Source Computer Vision Library, http://opencvlibrary.sourceforge.net/. Acesso em: Março 2011.

RABUZIN, K.; Baca, M.; Sjako, M. E-learning: biometrics as a security factor, Proceedings of the International Multi-Conference on Computing in the Global Information Technology, pp. 64-74, 2006.

ROMDHANI, S. Face Recognition Using Principal Component Analysis. University of Glasgow. Dissertação, 1996.

ROLIM, A. L.; Bezerra E. P. Um sistema de identificação automática de faces para um ambiente virtual de ensino e aprendizagem. In Companion Proceedings of the XIV Brazilian Symposium on Multimedia and the Web, 2008.

RUTHSCHILLNG, E. E. et al. A evolução dos ambientes de aprendizagem construtivistas. Disponível por http://penta.ufrgs.br/ luis/Ativ1/AmbApC.html, Acesso em 05 de julho. 2011.

STAN, Z. Li; Anil K. J. Handbook of Face Recognition, 2nd Edition, Springer, 2011.

TURK, M; Pentland A. Eigenfaces for recognition. Journal of Cognitive Neuroscience, 3(1):71\{86, 1991.

VADIVEL, A.; Majumdar A. K.; Sural S. Performance Comparison of Distance Metrics in Content-based Image Retrieval Applications, International Conference on Information Technology (CIT), Bhubaneswar, India, pp. 159-164, 2003. 
11

VIOLA, P. A.; Jones M. J. Robust real-time object detection, International Journal of Computer Vision, 57(2): 137-154, 2004. 